\title{
Subtipos de lectores RETRASAdos EN ESPAÑOL
}

\author{
SUBTYPES OF SUBJECTS WITH READING DISABILITIES \\ IN THE SPANISH LANGUAGE
}

\author{
Ariel Cuadro \\ Universidad Católica del Uruguay \\ Javier Marín \\ Universidad de Murcia (España)
}

\begin{abstract}
Resumen: Con el objetivo de identificar y caracterizar a subtipos de lectores retrasados en español (fonológicos y superficiales), se comparan 42 lectores retrasados de $4^{\circ}, 5^{\circ}$ y $6^{\circ}$ de primaria, con 90 lectores normales de igual grado escolar y con 78 lectores normales de menor grado escolar y nivel lector equivalente. Se analizó el rendimiento de los grupos en tareas que implican procesamiento fonológico o procesamiento ortográfico. Los resultados de someter los datos a un análisis de regresión, muestran variaciones en la distribución de los subtipos de lectores retrasados. Se aplica la idea de que la dislexia de tipo fonológico y superficial, se diferenciaría en el grado de severidad del desorden fonológico y en los recursos cognitivos disponibles para compensar el déficit. (Snowling, 2000). La transparencia de la lengua, así como la experiencia lectora, favorece el aprendizaje de las reglas de correspondencia grafema-fonema, mejorando la exactitud en la lectura de pseudopalabras.
\end{abstract}

Palabras claves: lectura, dificultades en lectura, dislexia, evaluación

\begin{abstract}
With the purpose of identifying and demonstrating the characteristics of dyslexic subtypes in the Spanish language (phonological and superficial dyslexics), 42 subjects with reading disabilities were assessed in the $4^{\text {th }}, 5^{\text {th }}$, and $6^{\text {th }}$ elementary grades as well as 90 subjects with normal reading ability in the same grades and 78 subjects with normal reading ability on a lower elementary grade but equivalent reading ability. Group performance was analyzed in tasks that involved phonological and orthographic processing. Using a regression analysis methodology we find some variations in the distribution of the subtypes of subjects with reading disabilities. The idea that the types of phonological and superficial dyslexia are different in severity degree in the phonological disorder and the cognitive resources available to compensate for it is applied (Snowling, 2000). The transparency of the language, as well as the reading experience, favors the learning of the grapheme-phoneme rule, improving the reading accuracy of non-words.
\end{abstract}

Keywords: reading, reading disability, dyslexia, assessment.

\section{INTRODUCCIÓN}

La lectura es una habilidad compleja que implica para ser dominada, una serie de procesos y subprocesos; algunos vinculados a la comprensión del lenguaje y otros más básicos y específicos como los que permiten el reconocimiento de palabras (procesamiento léxico y subléxico).

Los procesos de reconocimiento de palabra, son fundamentales para alcanzar una lectura diestra; y el desarrollo de la exactitud y de la 
velocidad de esto procesos facilitan el procesamiento exitoso en los niveles superiores (Perfetti, 1985, 1986).

A partir de la interpretación del modelo de "doble ruta", podemos diferenciar dos modos o procedimiento de reconocer una palabra: el léxico o directo (ruta léxica) y los subléxicos o indirectos (ruta fonológica). La vía léxica permite un reconocimiento inmediato de las palabraspartir de representaciones de esas palabrasalmacenadas previamente en nuestra memoria; algo que depende de la experiencia repetida con esas palabras escritas. La vía fonológica utiliza las reglas de correspondencia grafemafonema, ya que el sistema alfabético asigna a cada letra un sonido, para convertir los grafemas en representaciones de fonemas y así activar las formas fonológicas de las palabras.

Estos procesos no operan independientemente sino que son procesos que interactúan y se afectan mutuamente. Share $(1985,1999)$ incluso ha defendido la idea de que la recodificación fonológica funciona como un mecanismo de autoaprendizaje, permitiendo la adquisición de las representaciones ortográficas necesarias para un reconocimiento visual de las palabras rápido y autónomo. Cada decodificación correcta de una palabra no familia, da la oportunidad de adquirir la información ortográfica precisa de la palabra, que es la base para un reconocimiento eficaz de las palabras.

Al estudiar los sujetos que presentan dificultades en el reconocimiento de palabras se han podido diferenciar subtipos distintos de problemas (Castles y Coltheart, 1993; Seymour y MacGregor, 1984; Seymour y Evans, 1993; Manis, Seiddenberg, Doi, Mc Bride.Chang y Petersen, 1996; Genard 2000; Calvo 1999). Los llamados disléxicos fonológicos, con un déficit especialmente en la ruta fonológica valorada a través de la lectura de pseudopalabras y los disléxicos de superficie, la que es valorada a partir de la lectura de palabras irregulares o de pruebas de decisión léxica en el caso del español. Es posible también encontrar personas con dificultades en ambas vías, los disléxicos mixtos.

Los estudios comparativos entre disléxicos y lectores normales de la misma edad cronológica y entre disléxicos y lectores normales de menor edad e igual nivel lector (Castles y Coltheart, 1993; Manis et. al., 1996; Stanovich et al., 1997; Snowling et. al., 1998; Calvo, 1999 Genard, 2000; Bailey et al., 2004) han permitido una mayor caracterización entre ambos subtipos, si bien aún hay dudas sobre la naturaleza del deterioro. Mientras lo disléxicos de superficie se comportan de forma muy parecida a los lectores normales más jóvenes, lo que sugiere un retraso madurativo; los disléxicos fonológicos se presentan como una desviación en su desarrollo, en cuanto su rendimiento en palabras no familiares 0 pseudopalabras fue inferior a los normales de menor edad e igual nivel lector.

Así Castles y Coltheart (1993) a la hora de evaluar si existe un déficit relativo ${ }^{1}$ entre los disléxicos, en la ejecución de las tareas que evalúan la ruta fonológica (lectura de pseudopalabras) y las que valoran la ruta léxica (lectura de palabras irregulares) utilizaron una metodología de regresión en función de los datos de lectores normales igual edad cronológica. Definieron los subtipos a partir de los límites inferiores de la líneas de regresión que se establecen del análisis de los resultados de lectura de pseudopalabras sobre la lectura de palabras y de está última sobre la lectura de pseudopalabras. Concluyeron que los subtipos de dislexia fonológica y de superficie son bastante comunes de encontrar en los grupos de disléxicos evolutivos y que estos subtipos representan a diferencias en el funcionamiento de las dos vía de procesamiento lector.

Manis F., Seidenberg M., Doil, L., Mc BrideChang C. y Petersen A. (1996) replicaron el estudio de Castles y Coltheart (1993) aplicando la metodología regresión, comparando niños disléxicos y lectores normales, pero pareados no sólo por edad cronológica sino también por nivel lector, utilizando un grupo control de lectores normales más jóvenes. De este modo concluyen que los disléxicos fonológicos tienen un déficit específico en el proceso fonológico y los de superficie presentan un retraso más general en el reconocimiento de palabras. Resultados similares aparecen en el estudio de Bailey, Manis, Perdersen y Seidenberg (2004).

Stanovich, Siegel y Gottardo (1997) realizaron otro estudio comparativo de disléxicos con

${ }^{1}$ En un primer estudio comparan los resultados que obtienen 53 disléxicos en lectura de pseudopalabras y de palabras irregulares con los que tienen 56 lectores normales de la misma edad cronológica. Definen los subgrupos a partir del análisis de regresión aplicado a los resultados de los lectores normales en esas pruebas, en función de la edad cronológica. 
lectores normales de menor edad que los utilizados por Castles y Coltheart (1993) y Manis et al. (1996); y confirmaron que el grupo de disléxicos fonológicos tiene un mayor déficit que los lectores normales más jóvenes de similar nivel lector. Los disléxicos de superficie muestran más bien un retraso madurativo, el que podría explicarse por ser niños con problemas fonológicos moderados con poca exposición a las palabras impresas.

Genard (2000) utiliza la metodología señalada en los estudios anteriores pero en niños de habla francesa, que posee una ortografía más trasparente que el inglés ${ }^{2}$; además incluye las comparación de disléxicos con lectores normales de similar edad cronológica, pero controlando estadísticamente el nivel de comprensión lectora, como forma de abordar el problema de la desviación o retraso y el de la experiencia lectora. En su investigación aparecen diferencias con los trabajos de Castles y Coltheart (1993) y Manis et.al (1996), en cuanto a la proporción de disléxicos fonológicos y superficiales, habiendo mayor cantidad de estos últimos. Estas diferencias podrían explicarse por la mayor transparencia ortográfica del francés con respecto al inglés y por el énfasis en el uso de la correspondencia grafema-fonema.

Siguiendo la misma metodología Calvo (1999) 3 estudió los subtipos de dislexia en español y obtuvo resultados que se asemejan más a los estudios en inglés que a lo realizado por Genard (2000) en francés. Por otro lado su trabajo parece confirmar la idea de que los disléxicos fonológicos presentan un patrón desviado de lectura. Encontró además que los disléxicos de superficie son más lentos que el grupo fonológico en cuanto a la velocidad con la que realizan la lectura de palabras y pseudopalabras, debido posiblemente a que los superficiales tienen un procesamiento lector más analítico por lo que comenten menos errores a costo de mayor tiempo. Manis et al (1999) con una muestra de lectores iniciales ingleses, también encontraron que una característica importante de los disléxicos superficiales es su lentitud para leer.
Considerando el tema de las de las variaciones en la distribución de los perfiles disléxicos, los resultados de Calvo (1999) no se correspondería con la tesis de la transparencia del idioma (Genard, 2000) que señala que en lenguas trasparentes sería significativamente menor la proporción de disléxicos fonológicos. Más allá de las cuestiones metodológicas que Calvo (1999) plantea al analizar sus resultados, constituye, para este trabajo, un objetivo importante continuar en esta línea de investigación con nuevos datos en español; por las implicancias que tendría para el diagnóstico y la intervención..

Ahora bien esta consecuencia práctica de establecer subtipos de disléxicos no justifica por si mismo que existan dos o más categorías de disléxicos claramente delimitadas (Morais; Aaron et al., 1999). Podría haber un continuo entre un subgrupo y otro en que se identificarían casos extremos. Un continuo que se explica por la interdependencia y la sucesión, a través del desarrollo de la habilidad lectora, de los procesos de reconocimiento de palabra (Frith, 1985; MOrai, 1998). Para lo cual hay que considerar también la transparencia ortográfica de la lengua y el tipo de enseñanza. Siendo este otro de los objetivos que nos planteamos considerar y analizar en este estudio.

Asimismo en un trabajo como este, valorar las habilidades metafonológicas resulta ineludible, las investigaciones han comprobado la relación reciproca que hay entre el conocimiento de las habilidades metafonológicas y aprendizaje lector. El conocimiento metafonológico es una variable fundamental en la adquisición de la lectura y en la explicación de los trastornos lectores (Share y Satnovich, 1995).

\section{MÉTODO}

\section{Participantes}

La muestra de lectores normales y de lectores retrasados se obtuvo a partir de una población de 930 alumnos de $2^{\circ}$ a $6^{\circ}$ de Educación Primaria de tres centros privados de la ciudad

\footnotetext{
${ }^{2}$ La ortografía francesa es tan opaca como la inglesa desde el punto de vista de la escritura de palabras (v.g. la construcción de una representación ortográfica a partir de una fonológica) dado el alto número de transcripciones que no están determinadas por una regla. Sin embargo, desde el punto de vista de la lectura, el francés es una lengua mucho más transparente que el inglés, debido a un porcentaje mucho mayor de palabras escritas pueden ser correctamente pronunciadas atendiendo al conjunto de reglas ortográficas de la lengua francesa (Alegría y Mousty, 1994).

${ }^{3}$ Para le evaluación de ruta léxica utilizó una prueba de discriminación ortográfica, ya que en castellano no existen palabras irregulares.
} 
de Montevideo, de niveles socio-económicos medio bajo, medio y medio alto respectivamente. Se eligieron 150 lectores normales, 30 por grado escolar (15 varones y 15 mujeres) y 42 lectores retrasados de $4^{\circ}$ a $6^{\circ}$ año.

Los lectores normales se eligieron de acuerdo a los siguientes criterios:

- Tener un nivel intelectual medio o superior de acuerdo a la evaluación diagnóstica realizada en las instituciones al ingresar a ellas ${ }^{4}$.

- No presentar deficiencias sensoriales, ni padecer ni haber padecido patologías neurológicas conocidas.

- Obtener una puntuación media para su nivel en la prueba TECLE de eficacia lectora (Marín y Carrillo, 1999).

Los lectores retrasados se identificaron siguiendo estos criterios:

- Tener un nivel intelectual medio o superior de acuerdo a la evaluación diagnóstica realizada en las instituciones al ingresar a ella y obtener en la prueba de inteligencia Factor $\mathrm{G}$ (Cattell y Cattell, 1994) un C.I. igual o superior a 85.

- No presentar deficiencias sensoriales, ni padecer ni haber padecido patologías neurológicas conocidas.

- Obtener una puntuación por debajo de la media menos una desviación estándar en la prueba TECLE de eficacia lectora (Marín y Carrillo, 1999).

- Ser además confirmados como lectores retrasados por una maestra especializa$\mathrm{da}^{5}$ de nuestro medio, no pertenecientes a las instituciones a la que los alumnos pertenecen.

El rango de edad de los lectores normales fue de 7 a12 años y el de los lectores retrasados de 9 a 12 años.

\section{Materiales}

\section{Evaluación del coeficiente intelectual}

Se aplicó el Test de Factor $G$ de R. B. Cattell y A. K. S. Cattell (1994), Escala 2, Forma A.

\section{Evaluación del nivel lector}

Para la evaluación del nivel lector se utilizó la prueba TECLE de eficacia lectora (Marín y Carrillo, 1999), que ha demostrado su eficacia a la hora de evaluar el nivel lector (Calvo, 1999; Marín, Aveledo, Vera y Alegría, 2004; Cuadro, 2006).

\section{Evaluación de los componentes de la ha- bilidad lectora}

Lectura de palabras y pseudopalabras.

La prueba consiste en la lectura de tres listas, cada una de 30 palabras y pseudopalabras mezcladas. Algunas las investigaciones han mostrado que las estrategias utilizadas en lectura de palabras y pseudopalabras independientes son distintas (Cuetos, Rodríguez y Ruano, 1998). Las palabras y pseudopalabras fueron extraídas del test PROLEC (Cuetos, Rodríguez y Ruano, 1998), Algarabel, (1996) y Santos (1996). Se controló la proporción de palabras y de pseudopalabras y la complejidad ortográfica de las mismas. La primera lista contiene el $70 \%$ de palabras y el $30 \%$ de pseudopalabras de estructura ortográfica simple 6 . La segunda tiene el $30 \%$ de palabras y el $70 \%$ de pseudopalabras también con estructura ortográfica simple. La tercera contiene $30 \%$ palabras y $70 \%$ pseudopalabras con estructura ortográfica compleja.

La prueba se valora sumando los errores cometidos en las tres listas y considerando la suma de todos los tiempos (en segundos) invertidos en la lectura de las listas ${ }^{7}$. Si un sujeto

\footnotetext{
${ }^{4}$ Aunque se es conciente de que el nivel lector no depende del $\mathrm{Cl}$ y por tanto su uso no es aconsejable (Share y Stanovich, 1984; Morais 1998) lo empleamos por consistencia con otros estudios que tradicionalmente lo han considerado, de igual modo que el DSM IV lo sigue incluyendo dentro de sus criterios diagnósticos

${ }^{5}$ Las maestras especializadas, son maestras que tienen formación en dificultades de aprendizaje y son en nuestros país por lo general las que evalúan y hacen la intervención a los niños con trastornos en lectura

${ }^{6}$ Las cadenas con estructura ortográfica simple están constituídas a partir de sílabas V (Voca) o CV (consonantevocal). Las cadenas con estructura compleja presentaban al menos una sílaba compleja de tipo CCV,CVC,VC, etc. ${ }^{7}$ Estas medidas han sido tomadas manualmente tanto sobre las respuestas correctas como las incorrectas y están incluidos los tiempos de cambiar de un ítem a otro, las dudas y las autocorrecciones. Se ha demostrado que los tiempos relevados por ítems en computadoras presentas una correlación elevada con los tiempos tomados manualmente (Genard, 1999).
} 
leía una palabra o pseudopalabra en forma equivocada pero espontáneamente la rectificaba, se puntuaba como acierto.

\section{Discriminación ortográfica}

Para evaluar el mecanismo de acceso ortográfico directo para el reconocimiento de palabras se construyo esta prueba, que para su correcta ejecución requiere que los sujetos dispongan de una representación ortográfica precisa de las palabras.

La prueba está formada por 20 palabras y 20 pseudohomófonos de esas palabras. Se seleccionaron palabras familiares y de baja dificultad ortográfica (igual o inferior a 10 según el Vocabulario Básico Ortográfico de Mensanza (1990); que contenían algún grafema inconsistente: $\mathrm{v} / \mathrm{b}, \mathrm{s} / \mathrm{c}, \mathrm{y} / \mathrm{ll}, \mathrm{j} / \mathrm{g}$ y $\mathrm{h}$. Los pseudohomófonos se construyeron sustituyendo el grafema correcto por su equivalente fonológico o se suprimía o añadía la letra " $h$ ".

Para la corrección se consideró el número de errores cometidos y el tiempo invertido (en segundos) para completar todos los ítems de la prueba.

\section{Evaluación de las habilidades metafonológicas}

Prueba de omisión de primera sílaba

Se utilizaron los ítems de la prueba elaborada por Asensio (1989) y utilizadas por Santos (1996). La prueba se compone de seis palabras y seis pseudopalabras y la forma de presentación al niño es sólo oral.

\section{Prueba de omisión de primer fonema}

Se emplearon los ítems de la prueba diseñada por Asensio (1989) y utilizados por Santos (1996). La prueba se compone de ocho palabras y ocho pseudopalabras.

\section{Procedimiento}

Se aplicó primero la prueba TECLE de eficacia lectora, en forma colectiva en las aulas de cada grupo de clase elegida. A partir de los resultados de la prueba TECLE de eficacia lectora se seleccionó el grupo de lectores normales y el de lectores retrasados. Se les aplicó dividiéndolos por grado escolar, en cada colegio, la prueba de Factor "g" de Cattell y Cattell (1994) y en el caso de los lectores retrasados se solicitó a una maestra especializada que evaluara en forma individual sus niveles de lectura. En el caso de los buenos lectores, así como de los lectores retrasados, se solicitaron los informes de ingresos a la institución, en cuanto a nivel intelectual, historia evolutiva y aspectos familiares.

De este modo y siguiendo los criterios de exclusión establecidos se eligieron los sujetos de la muestra, los que fueron evaluados individualmente en espacios destinado por cada colegio. Se aplicaron en una misma sesión las pruebas de evaluación de la habilidad lectora y de las habilidades metafonológicas.

En un primer estudio se compararon los resultados de los subtipos de lectores retrasados $(\mathrm{N}=42)$, identificados a partir del análisis de regresión usado por Castles y Coltheart (1993), entre sí y con los lectores normales $(\mathrm{N}=90)$ de igual grado escolar (CG); en las pruebas de lectura de pseudopalabras y de decisión ortográfica (considerando número de errores y tiempo) y de habilidades metafonológicas.

En un segundo estudio se compararon los resultados de las pruebas de los subtipos de lectores retrasados $(\mathrm{N}=42)$ identificados a partir del análisis de regresión usado por Castles y Coltheart (1993), entre sí y con los lectores normales $(\mathrm{N}=78)$, de igual nivel lector $(\mathrm{CL})$.

En un tercer estudio se compararon los resultados de los subtipos de lectores retrasados $(\mathrm{N}=42)$ identificados a partir del análisis de regresión usado por Castles y Coltheart (1993), entre sí y con los lectores normales $(\mathrm{N}=90)$, de igual grado escolar, controlando estadísticamente el nivel lector.

\section{RESULTADOS}

Para los estudios 1 y 2 se siguió la metodología de Castles y Coltheart (1993), para identificar a los subtipos de lectores retrasados, se calculó la ecuación de regresión en la tarea de pseudopalabras sobre la de decisión ortográfica realizada por los lectores normales de igual grado escolar (CG) y los igual nivel lector (NL) respectivamente y se estableció un intervalo de confianza del $90 \%$ en torno a la línea de regresión; luego se calculó la relación opuesta, es decir discriminación ortográfica actuó como criterio de predicción sobre la puntuación en pseudopalabras.

Para la comparación entre los distintos subgrupos de lectores retrasados y el grupo de lectores normales de igual grado escolar y de 
igual nivel lector en las tareas realizadas utilizamos pruebas no paramétricas ya que las variables elegidas no cumplen con los criterios necesarios de (homogeneidad de la varianza, análisis de normalidad y nivel de medición) para aplicar pruebas paramétricas.

Estudio 1: Subtipos de lectores retrasados en relación con lectores normales del mismo grado escolar

En la Figura 1 se presenta la distribución de la muestra de los lectores retrasados (LR) que se agrupan a partir de los ejes de corte dados por los límites superiores establecidos a partir de las líneas de regresión definida en los lectores normales (CG). Los resultados de la agrupación realizada aparecen en la Tabla 1.

En la Tabla 2 se presentan los resultados de los subgrupos de lectores retrasados establecidos y del grupo de lectores normales en las diferentes pruebas aplicadas de evaluación de la lectura y pruebas metafonológicas
La prueba de Kruskall-Wallis, con la cual se comparan las medias de los cinco grupos (control, moderado, superficial, fonológico y severo) muestran diferencias significativas en todas las tareas menos en omisión del primer fonema.

Las comparaciones posteriores entre pares de grupos se realizaron con la prueba de $U$ Mann-Whitney, acompañada de la corrección de Bonferroni para controlar la tasa de error.

En la prueba de eficacia lectora (TECLE), de las cinco comparaciones posibles, entre el grupo control y los cuatros grupos con retraso lector, encontramos, que los del grupo control (CG), rindieron significativamente mejor que los alumnos pertenecientes a los cuatro subgrupos evaluados con retraso lector (moderado, superficial, fonológico y severo), puesto que el nivel crítico es $p=0.00$. En esta prueba, los cuatro grupos de lectores retrasados no difieren entre sí.

En lectura de pseudopalabras, no se encontraron diferencias significativas entre el grupo control y moderados $(p=0,22)$ y control y su-

Fig. 1. Identificación de los subtipos de lectores retrasados a partir de los ejes de corte dados por los límites superiores establecidos a partir de las líneas de regresión definida en los lectores normales (CG).

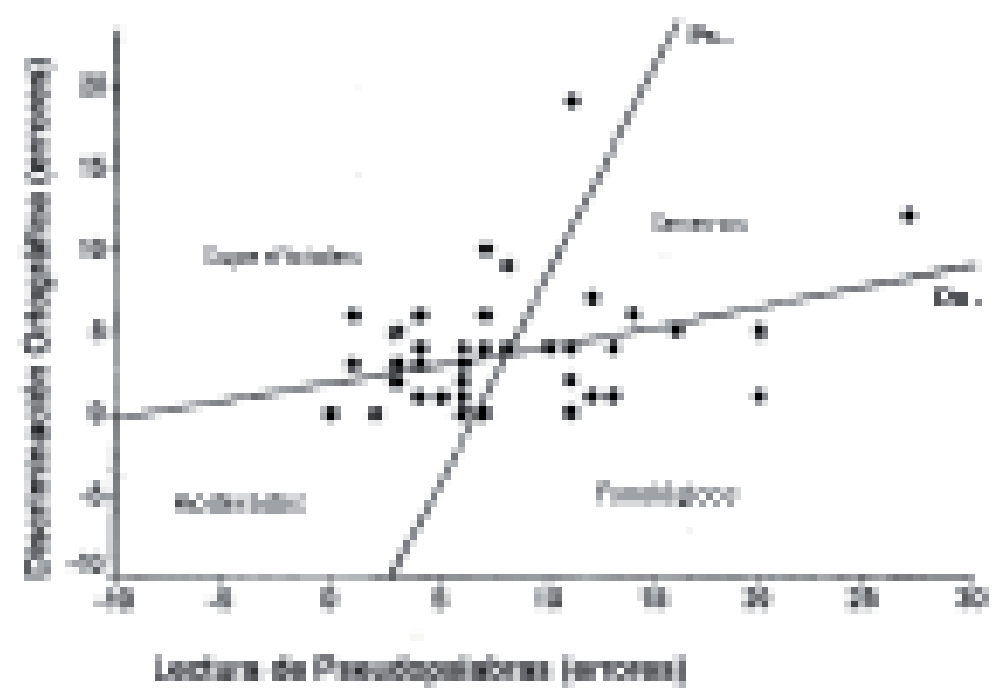

Tabla 1. Número y porcentaje de lectores retrasados que integran los subtipos definidos para lectores retrasados y lectores normales de igual grado escolar

\begin{tabular}{lcc}
\hline & Número de LR & Porcentaje de LR \\
\hline Severos & 3 & 7,42 \\
Fonológicos & 13 & 30,9 \\
Superficiales & 15 & 35,7 \\
Moderados & 11 & 26,1 \\
\hline
\end{tabular}


Tabla 2: Media y desviación estándar de las puntuaciones directas en las pruebas de eficacia lectora, lectura de pseudopalabras, discriminación ortográfica (considerando número de errores y de tiempo) y pruebas metafonológicas (omisión de sílaba inicial y omisión de fonema inicial) para los subgrupos de lectores retrasados y lectores normales de igual grado escolar.

\begin{tabular}{|c|c|c|c|c|c|}
\hline & $\begin{array}{c}\text { Fonológicos } \\
\text { Med. (sd) }\end{array}$ & $\begin{array}{c}\text { Superficiales } \\
\text { Med. (ds) }\end{array}$ & $\begin{array}{l}\text { Severos } \\
\text { Med.(ds) }\end{array}$ & $\begin{array}{l}\text { Moderados } \\
\text { Med.(ds) }\end{array}$ & $\begin{array}{c}\text { (CG) } \\
\text { Med. (ds) }\end{array}$ \\
\hline Nivel Lector & $15,38(4,27)$ & $16,93(5,68)$ & $11,00(4,36)$ & $17,73(4,31)$ & $32,08(5.20)$ \\
\hline $\begin{array}{l}\text { Lectura } \\
\text { pseudopalabras }\end{array}$ & $12,69(3,83)$ & $5,20(2,80)$ & $17,66(8,14)$ & $4,27(2,05)$ & $3,8(3,14)$ \\
\hline $\begin{array}{l}\text { Tiempo lectura } \\
\text { pseudopalabras }\end{array}$ & $216,38(93,07)$ & $170,46(95,87)$ & $514,66(321,01)$ & $137,45(43,42)$ & $107,98(21,20)$ \\
\hline $\begin{array}{l}\text { Discriminación } \\
\text { ortográfica }\end{array}$ & $2,54(1,85)$ & $5,93(4,20)$ & $8,33(3,21)$ & $1,27(1,01)$ & $1,04(1,72)$ \\
\hline $\begin{array}{l}\text { Tiempo discr. } \\
\text { ortográfica }\end{array}$ & $130,92(55,94)$ & $161,93(92,98)$ & $278,33(162,61)$ & $101,09(33,19)$ & $52,34(14,45)$ \\
\hline $\begin{array}{l}\text { Omisión de sílaba } \\
\text { inicial }\end{array}$ & $1,46(1,51)$ & $0,47(1,30)$ & $2,00(2,00)$ & $0,82(1,83)$ & $0,24(0,53)$ \\
\hline $\begin{array}{l}\text { Omisión de } \\
\text { fonema inicial }\end{array}$ & $1,46(1,85)$ & $1,07(1,58)$ & $4,00(3,61)$ & $0.64(0.81)$ & $0,59(0,90)$ \\
\hline
\end{tabular}

perficial $(p=0,05)$; pero los del grupo control tienen un mejor desempeño que los grupos con retraso lector: fonológico $(p=0.00)$ y severo $(p=0.00)$. Analizando las comparaciones posibles entre los grupos con retraso lector encontramos que el grupo moderado no difiere significativamente con el grupo de superficie $(p=0,35)$, pero si son significativamente mejores que el fonológico y el severo $(p<0,005)$. Se encontró también que los alumnos del subtipo superficial rinden mejor que los del grupo fonológico y también que los del severo; el nivel crítico en estas comparaciones es 0,00 y 0,002 respectivamente. No hay diferencias significativas entre fonológicos y severos $(p=0,14)$

Cuando consideramos el tiempo en lectura de pseudopalabras, el grupo control no difiere con el subtipo moderado $(p=0,03)$, pero si es significativamente más rápido que los grupos de retraso lector: superficial, fonológico y severo, con una $p=0,00$. El grupo moderado es también más rápido que el fonológico y severo $(p=0.00)$ pero no del superficial $(p=0,28)$. Este último no difiere significativamente del fonológico $(p=0,13)$ ni del severo $(p=0,10)$. Como tampoco hay diferencias entre el fonológico y el severo $(p=0,025)$.

En la tarea de discriminación ortográfica, los lectores del grupo control se desempeñan mejor que los lectores retrasados del subtipo superficial $(p=0,00)$, fonológico $(p=0,002)$ y severo $(p=0,00)$. Encontramos también que del grupo de moderados son mejores que los de superficie $(p=0,00)$ y severo $(p=0,005)$. El subgrupo de fonológico es más eficiente en esta tarea que el severo $(p=0,00)$.

En la variable tiempo de discriminación ortográfica, si comparamos el grupo control (GE) con los cuatros grupos con retraso lector, encontramos que el grupo control rinde significativamente mejor que los alumnos pertenecientes a los cuatro subgrupos evaluados con retraso lector (moderado, superficial, fonológico y severo), puesto que el nivel crítico en las cuatro comparaciones es $p=0,00$. Asimismo el subtipo moderado es más rápido que el de superficie $(p=0,03)$ y que el severo $(p=0.01)$. No hay diferencias entre el de superficie y el fonológico, ni es entre estos y el severo.

En la tareas de omisión de sílaba inicial; se encontraron diferencias significativa a favor del grupo control (GE) en relación al subtipo fonológico $(p=0.00)$, no así con el moderado $(p=0,47)$, el superficial $(p=1,00)$ y el severo $(p=0,09)$. Analizando las comparaciones posibles entre los grupos de retraso lector no encontramos diferencias significativas.

En la tarea de omisión de fonema inicial; no se encontraron diferencias significativas menores (con un nivel de significación del 0,005 ) entre los grupos de control y grupos con retraso lector, ni entre los grupos de lectores retrasados entre sí 
Estudio 2: Subtipos de lectores retrasados en relación con lectores normales del mismo nivel lector.

En la Figura 2 se presentan la distribución la muestra de los lectores retrasados (LR) a partir de las líneas de regresión definidas en los lectores normales (CL). Los resultados de la agrupación realizada aparecen en la Tabla 3.

En la Tabla 4 se presentan los resultados de los subgrupos de lectores retrasados establecidos y del grupo de lectores normales en las diferentes pruebas aplicadas de evaluación de la lectura y pruebas metafonológicas.

La prueba de Kruskall-Wallis, con la cual se comparan las medias de los cuatro grupos (control, moderado, superficial, fonológico) muestran diferencias significativas en todas las tareas menos en omisión del primer fonema y nivel lec- tor. Las comparaciones posteriores entre pares de grupos, con la prueba U Mann-Whitney acompañada de la corrección de Bonferroni muestran:

En lectura de pseudopalabras, no se encontró diferencias significativas entre el grupo control $(C L)$ y el moderado $(p=0,13)$ ni entre el grupo control $(C L)$ y el subtipo superficial $(p=0,25)$. Los lectores normales del grupo control rinden mejor los alumnos del subgrupo fonológico $(p=0,00)$, y estos últimos son menos eficientes que los del grupo moderado $(p=0,00)$. No existen diferencias significativas entre el grupo superficial y el fonológico $(p=0,11)$; ni entre el moderado y superficial $(p=0,44)$.

En la tarea de tiempo de lectura de pseudopalabra los del grupo control $(C L)$ no difieren con los del moderado $(p=0,37)$ ni con los de superficie $(p=0,41)$. En cambio si son más

Figura 2: Identificación de los subtipos de lectores retrasados a partir de los ejes de corte dados por los límites superiores establecidos a partir de las líneas de regresión definidas en los lectores normales $(C L)$

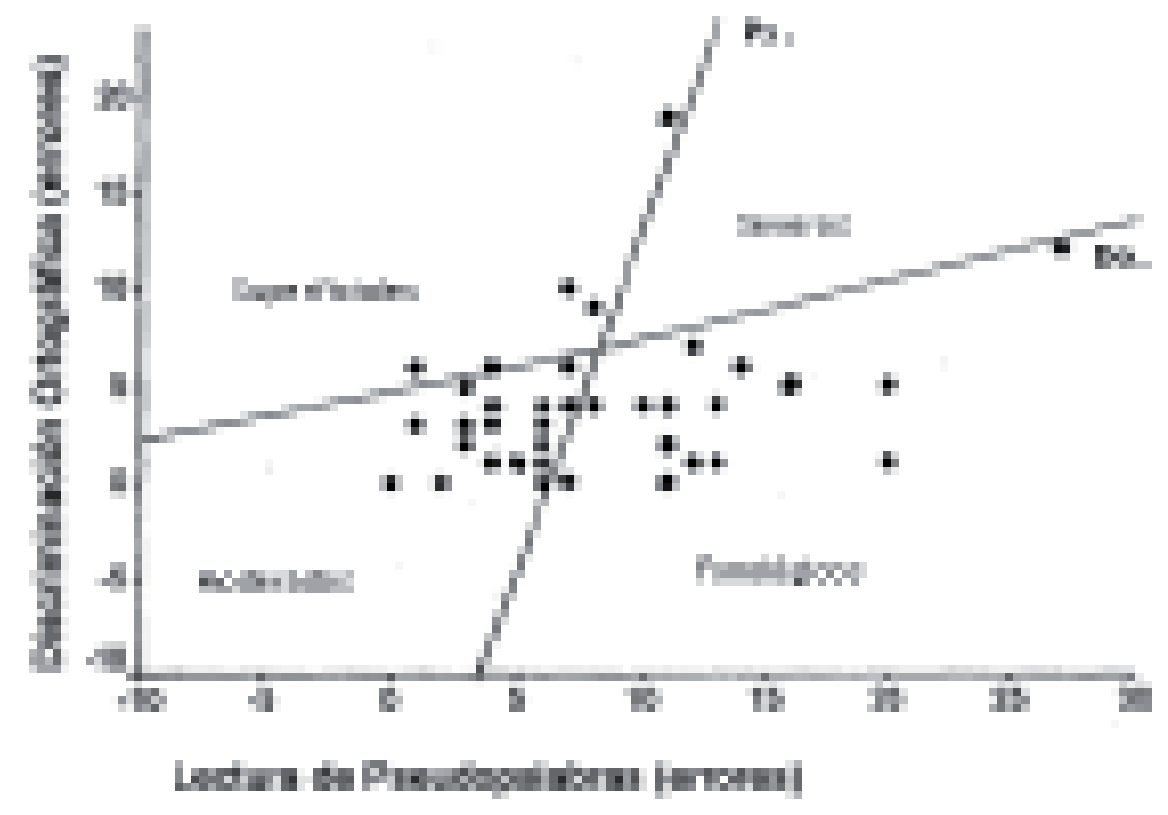

Tabla 3: Número y porcentaje de lectores retrasados que integran los subtipos definidos para lectores retrasados y lectores normales de igual nivel lector

\begin{tabular}{lcc}
\hline & Número de LR & Porcentaje de LR \\
\hline Severos & 0 & 0 \\
Fonológicos & 15 & 35,7 \\
Superficiales & 5 & 11,9 \\
Moderados & 22 & 52,3 \\
\hline
\end{tabular}


Tabla 4: Media y desviación estándar de las puntuaciones directas en las pruebas de eficacia lectora, lectura de pseudopalabras, discriminación ortográfica (considerando número de errores y de tiempo) y pruebas metafonológicas (omisión de sílaba inicial y omisión de fonema inicial)para los subgrupos de lectores retrasados y lectores normales de igual nivel lector.

\begin{tabular}{lcccc}
\hline & Fonológicos & Superficiales & Moderados & $(\mathrm{CL})$ \\
& Med. (sd) & Med. (ds) & Med.(ds) & Med. (ds) \\
\hline Nivel lector & $14,53(4,66)$ & $15,20(7,66)$ & $17,64(4,29)$ & $16,01(5,73)$ \\
Lectura pseudopalabras & $13,26(5,77)$ & $6,20(3,83)$ & $5,13(2,76)$ & $4,55(3,61)$ \\
Tiempo.lec. pseudopal. & $276,06(193,5)$ & $146,0(54,09)$ & $161,59(82,95)$ & $163,25(51,92)$ \\
Discriminación ortográfica & $3,73(3,17)$ & $10,0(5,34)$ & $2,50(1,63)$ & $3,35(2,71)$ \\
Tiempo discr. ortográfica & $165,6(94,78)$ & $204,0(140,22)$ & $117,0(52,76)$ & $106,54(43,48)$ \\
Omisión sílaba inicial & $1,60(1,59)$ & $1,0(2,24)$ & $0,55(1,34)$ & $0,69(1,05)$ \\
Omisión fonema inicial & $2,07(2,37)$ & $1,80(2,05)$ & $0,64(1,0)$ & $0,73(1,05)$ \\
\hline
\end{tabular}

rápidos que los fonológicos $(p=0,004)$. No difieren el grupo moderado con el grupo de superficie $(p=0,786)$ ni con el fonológico $(p=0,01)$. Tampoco existen diferencias significativas entre el superficial y el fonológico $(p=0,066)$.

En la tarea de discriminación ortográfica, no hay diferencias significativas en la comparación del grupo control (CL) con el grupo moderado $(p=0,3)$, ni con el fonológico $(p=0,06)$. Si se desempeñan mejor que los del subtipo de superficie $(p=0,00)$, así como los del grupo moderado son mejores que los superficiales $(p=0,00)$ y estos últimos peores que los fonológicos $(p=0,005)$. No existen diferencias significativas entre el subtipo moderado y el fonológico $(p=0,092)$.

En la variable tiempo de discriminación ortográfica, el grupo control $(\mathrm{CL})$ no difiere con el subtipo moderado $(p=0,42)$ ni con el de superficie $(p=0,083)$. Tampoco existen diferencias significativas entre el superficial y el fonológico $(p=0,55)$; ni entre el grupo moderado y los grupos superficial $(p=0,165)$ y fonológicos $(p=0,092)$. El grupo $C L$ si es significativamente más rápido que el subtipo fonológico $(p=0,00)$.

En las tareas de omisión de sílaba inicial, las comparaciones del grupo control $(\mathrm{CL})$ con el subtipo moderado dio un nivel crítico bilateral de 0,17 , con el superficial de 0,55 y con el fonológico de 0,02 . No existen tampoco diferencias significativas entre el grupo moderado y los grupos superficial $(p=0,92)$ y fonológicos $(p=0,02)$; ni entre el superficial y el fonológico $(p=0,26)$.
En la prueba de omisión de primer fonema, no se encontraron valores críticos menores a 0,008 en todas las comparaciones de los grupos, por lo tanto no existen diferencias significativas entre ellos.

Estudio 3: Subtipos de lectores retrasados en relación con lectores normales del mismo grado escolar, controlando estadísticamente el nivel lector.

Siguiendo las propuestas de Genard (2000) y Calvo (1999), en este estudio identificamos los subtipos de lectores retrasados a partir de la comparación con los resultados de las pruebas de lectura de pseudopalabras y de discriminación ortográficas de los lectores normales de igual grado escolar, una vez que se controló estadísticamente el efecto del nivel lector. El efecto del nivel lector está dado porque existe una relación lineal significativa entre las puntuaciones de la prueba de eficacia lectora y las de lectura de pseudopalabras ( $14,7 \%$ de la varianza explicada) y discriminación ortográfica $(32,2 \%$ de la varianza explicada).

Las puntuaciones residuales del grupo control (GL) indican el nivel de ejecución de los lectores normales en las tareas de lectura de pseudopalabras y de discriminación ortográfica independientemente del nivel lector.

En la figura 3 se presenta las distribución de la muestra de los lectores retrasados que, al igual que en los estudios anteriores, se agrupan a partir de los ejes de corte dados por los límites superiores establecidos por las 
Figura 3. Identificación de los subtipos de lectores retrasados a partir de los ejes de corte dados por los límites superiores establecidos por las líneas de regresión, definidas por las puntuaciones residuales en el grupo CG.

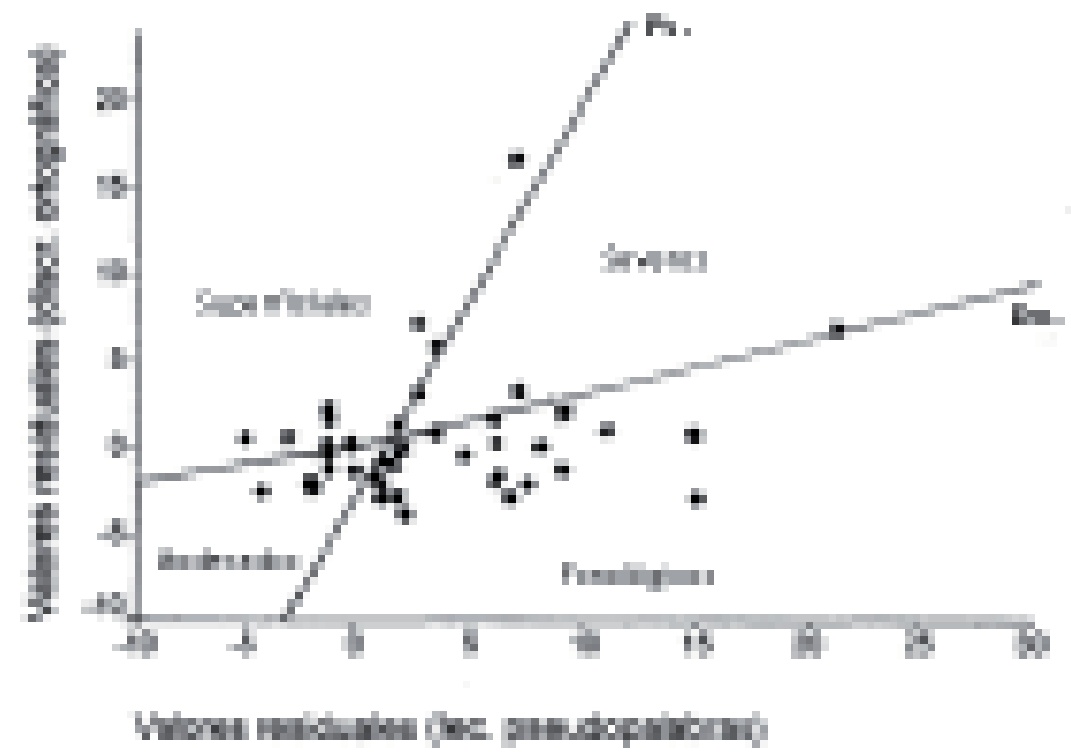

Tabla 5: Número y porcentaje de lectores retrasados que integran los subtipos definidos por lectores retrasados y lectores normales de igual grado escolar independiente del nivel lector

\begin{tabular}{lcc}
\hline & Número de LR & Porcentaje de LR \\
\hline Severos & 3 & 7,1 \\
Fonológicos & 24 & 57,1 \\
Superficiales & 10 & 23,8 \\
Moderados & 5 & 11,9 \\
\hline
\end{tabular}

líneas de regresión, en este caso definidas por las puntuaciones residuales. Los resultados de la agrupación realizada se presentan en la tabla 6.

En la tabla 6 se presentan los resultados de los subgrupos de lectores retrasados establecidos y del grupo de lectores normales (GL) en las diferentes pruebas aplicadas de evaluación de la lectura y pruebas metafonológicas

La prueba de Kruskall-Wallis, con la cual se comparan las medias de los cinco grupos (control, moderado, severo, superficial, fonológico) muestran diferencias significativas en todas las tareas menos en omisión del primer fonema.

Los análisis posteriores con la prueba U Mann -Whitney, acompañada de la corrección de Bonferroni muestran los siguientes resultados:

En la prueba de eficacia lectora (TECLE), los del grupo control GL rindieron significativamente mejor que los alumnos per- tenecientes a los cuatro subgrupos evaluados con retraso lector, puesto que el nivel crítico $(0,00)$ en todas las comparaciones. En esta prueba, los cuatro grupos de lectores retrasados no difieren entre sí.

En la prueba de lectura de pseudopalabras, no se encontraron diferencias significativas entre el grupo control $(G L)$ y moderado $(p=0,36)$ y el grupo control $(G L)$ y superficial $(p=0,26)$; pero sí se encontraron diferencias significativas a favor del GL con los grupos con retraso lector: fonológico $(p=0.00)$ y severo $(p=0,00)$. Analizando las comparaciones posibles entre los grupos con retraso lector, encontramos que el subtipo moderado no difiere significativamente con el grupo de superficie $(p=0,16)$, y lo hace moderadamente con los severos $(p=0,03)$; sí se desempeñan mejor que los fonológicos $(p=0,00)$. Se encontró también que los del subtipo de superficie rinden mejor que los 
Tabla 6: Media y desviación estándar de las puntuaciones directas en las pruebas de eficacia lectora, lectura de pseudopalabras, discriminación ortográfica (considerando número de errores y de tiempo) y pruebas metafonológicas (omisión de sílaba inicial y omisión de fonema inicial)para los subgrupos de lectores retrasados y lectores normales igual grado escolar, controlando estadísticamente el nivel lector.

\begin{tabular}{|c|c|c|c|c|c|}
\hline & $\begin{array}{c}\text { Fonológicos } \\
\text { Med. (sd) }\end{array}$ & $\begin{array}{l}\text { Superficiales } \\
\text { Med. (ds) }\end{array}$ & $\begin{array}{l}\text { Moderado } \\
\text { Med.(ds) }\end{array}$ & $\begin{array}{l}\text { Severos } \\
\text { Med. (ds) }\end{array}$ & $\begin{array}{c}\text { (GL) } \\
\text { Med. (ds) }\end{array}$ \\
\hline Nivel lector & $15,75(4,13)$ & $18,10(6,01)$ & $16,40(5,64)$ & $13,67(7,51)$ & $32,08(9,98)$ \\
\hline $\begin{array}{l}\text { Lectura } \\
\text { pseudopalabras }\end{array}$ & $9,87(4,55)$ & $4,9(3,24)$ & $2,4(1,51)$ & $15,0(10,81)$ & $3,8(3,14)$ \\
\hline $\begin{array}{l}\text { Tiempo lec. } \\
\text { pseudopalabras }\end{array}$ & $207,25(115,69)$ & $140,20(45,84)$ & $170,20(38,83)$ & $399,66(400,25)$ & $107,98(21,20)$ \\
\hline $\begin{array}{l}\text { Discriminación } \\
\text { ortográfica }\end{array}$ & $2,5(1,77)$ & $7,0(4,85)$ & $1,6(1,67)$ & $7,67(4,04)$ & $1,04(1,72)$ \\
\hline $\begin{array}{l}\text { Tiempo discr. } \\
\text { ortográfica. }\end{array}$ & $128,88(59,97)$ & $155,50(108,93)$ & $141,6(48,10)$ & $240,67(187,40)$ & $52,34(14,45)$ \\
\hline $\begin{array}{l}\text { Omisión sílaba } \\
\text { Inicial }\end{array}$ & $1,17(4,13)$ & $0,60(1,58)$ & $0,20(0,45)$ & $2,0(2,0)$ & $0,24(0,53)$ \\
\hline $\begin{array}{l}\text { Omisión fonema } \\
\text { inicial }\end{array}$ & $1,29(1,57)$ & $1,0(1,63)$ & $0,20(0,45)$ & $4,0(3,61)$ & $0,59(0,90)$ \\
\hline
\end{tabular}

fonológicos, con una $p=0,00$. No hay diferencias significativas entre los superficiales y los severos $(p=0,07)$ y los fonológicos y severos $(p=0,43)$.

Cuando consideramos el tiempo en lectura de pseudopalabras, el grupo control GL presenta diferencias moderamadamente significativas con los superficiales $(p=0,02)$ y con los severo $(p=0,03)$; es más rápido que de los grupos fonológico y moderados con una $p=0,00$ y $p=0,001$ respectivamente. El grupo de moderados no difiere del de fonológicos $(p=0,84)$, ni del de severo $(p=0,57)$, ni del de superficiales $(p=0,20)$. Este último no difiere significativamente del fonológico $(p=0,12)$, ni del severo $(p=0,11)$. Como tampoco hay diferencias entre el fonológico y el severo $(p=0,35)$.

En la tarea de discriminación ortográfica, el grupo control GL es más eficiente que los lectores retrasados del tipo superficial $(p=0,00)$, fonológico $(p=0,00)$ y severo $(p=0,00)$. Encontramos también que los superficiales son peores que los del subtipo moderado $(p=0,005)$ y los fonológicos $(p=0,001)$. No hay diferencias significativas entre los moderados con el grupo $\mathrm{GL}(p=0,351)$, ni con los fonológico $(p=0,352)$ y los severos $(p=0,036)$; tampoco difieren los severos con los fonológicos $(p=0,16)$, ni con los superficiales $(p=0,573)$.

En la variable tiempo de discriminación ortográfica, si comparamos el grupo control (GL) con los cuatros grupos con retraso lector, encontramos que el grupo GL rinde significativamente mejor que los alumnos pertenecientes a los cuatro subgrupos evaluados con retraso lector, puesto que el nivel crítico en las cuatro comparaciones es $p=0,00$. Los subtipos de retrasados no difieren entre sí.

En la tarea de omisión de sílaba inicial; los alumnos del grupo control (GL) se desempeñan significativamente mejor que los del subtipo fonológico $(p=0.001)$, no así con los del moderado $(p=0,934)$, del superficial $(p=0,953)$ y del severo $(p=0,09)$. Analizando las comparaciones posibles entre los grupos de retraso lector no encontramos diferencias significativas.

En la prueba de omisión del primer fonema; no se encontraron diferencias significativas menores a 0,005 entre el grupo de control (GL) y los grupos con retraso lector, ni entre los grupos con retraso lector entre sí.

\section{DISCUSIÓN Y CONCLUSIONES}

En estos estudios hemos definidos los subtipos de lectores retrasados en función de considerar la relación entre el procesamiento fonológico y ortográfico; los resultados de los mismos confirman, en un número importante, la existencia de subtipos de lectores retrasados del tipo fonológico y superficial, con sus perfiles característicos. Los superficiales obtienen mejores resultados en las tareas de lec- 
turas de pseudopalabras que en las de discriminación ortográfica y los fonológicos al contrario.

En la tabla 7 se presentan los porcentajes de los estudiantes de los grupos fonológicos y superficiales en las tres comparaciones realizadas, usando medidas relacionadas: lectores retrasados en relación con un grupo control de igual grado escolar (CG); lectores retrasados con un grupo control de igual nivel lector (NL); y los lectores retrasados en comparación con lectores normales de igual grado escolar, igualados en nivel lector (CGL).

En la primera de las comparaciones es decir entre lectores retrasados y lectores normales de igual grado escolar, hemos identificado un número algo mayor de disléxicos superficiales $(35,7 \%)$ que fonológicos $(30,9 \%)$. El que aparezca una proporción tan importante de disléxicos superficiales sugiere la idea de que muchos de los lectores retrasados con experiencia lectora, en lengua castellana, presentan un buen nivel de dominio de las reglas de correspondencia grafema-fonema y hacen uso de ello. Pero al mismo tiempo esto limitaría los recursos cognitivos necesarios (Manis et al, 1996) para almacenar las representaciones ortográficas específicas de las palabras, que permite su reconocimiento directo.

Este dominio en la utilización de la reglas correspondencia grafema-fonema estaría asociado a la transparencia de la lengua, ya que se facilitaría el aprendizaje de la reglas de correspondencia grafema-fonema. Los estudios con disléxicos de lengua alemana (Wimmer, 1993, 1996) indican que el déficit fonológico que los caracteriza no impide, por lo simple de su sistema alfabético, que estos niños adquirieran la competencia en la decodificación de las palabras a través de las reglas de conversión grafema-fonema. Genard (2000) trabajando con disléxicos francófonos, una lengua menos transparente que la nuestra, encontró también una proporción mucho mayor de disléxicos superficiales que fonológicos y atribuyo esta diferen- cia a la mayor transparencia del francés con respecto al inglés, además del tipo de instrucción (método fónico) que estos estudiantes habían recibido.

Si analizamos a los subtipos de lectores retrasados en relación con lectores normales más jóvenes aumenta la diferencia en la proporción de fonológicos $(35,7 \%)$ respecto a los superficiales $(11,9 \%)$. Los resultados muestran que desaparecen los severos, aumenta algo los fonológicos (dos casos) y disminuyen significativamente los superficiales; 10 de estos últimos pasan a formar parte de los moderados, que alcanzan un $52,3 \%$. Las diferencias encontradas entre los números de lectores retrasados del tipo fonológico y superficial, si consideramos los grupos controles de grado escolar y de nivel lector parecen indicar que los disléxicos fonológicos presentan un déficit específico en el procesamiento fonológico. Los superficiales por su parte se asimilan mejor a un retraso más general en el reconocimiento de palabras, en tanto no se diferencian en tareas fonológicas con los lectores normales más jóvenes y tienden a disminuir significativamente en número en este tipo de comparaciones. Estudios en diversas lenguas (Genard, 2000; Calvo, 1999; Manis et al. 1996; Stanovich et al, 1997; Snowling et al. 1998; Bailey et. al. 2004) revelan resultados muy similares a los expuestos.

Así por ejemplo Manis et al.(1996) encontró que los disléxicos fonológicos identificados en el análisis con lectores normales de similar edad, seguían presentado ese perfil cuando se los comparaba con lectores normales más jóvenes de similar nivel lector. Mientras los de superficie dejaban en su mayoría de pertenecer a este subtipo cuando de los comparaba con los lectores más jóvenes; los que le permite suponer que los disléxicos de superficie presentarían mas bien un retraso para leer respecto a su edad cronológica. Manis et al. (1996) atribuye este retraso a una limitación de los recursos

Tabla 7. Porcentaje de estudiantes de los grupos fonológicos y superficiales en todas las comparaciones realizadas.

\begin{tabular}{lccc}
\hline & LR - CG & LR-CL & LR-CGL \\
\hline Fonológicos & $30,9 \%$ & $35,7 \%$ & $57,1 \%$ \\
Superficiales & $35,7 \%$ & $11,9 \%$ & $23,8 \%$ \\
\hline
\end{tabular}


cognitivos. Stanovich et al. (1997) utilizando también el análisis de regresión propuesto por Castles y Coltheart (1993), con disléxicos y lectores normales equiparados en nivel lector, encontraron que los resultado confirman los hallazgos de Manis et al. (1996), por lo que creen que la dislexia de superficie se daría en sujetos con problemas fonológicos moderados pero sin experiencia lectora; por lo que no han logrado formar representaciones ortográficas consistentes de las palabras escritas.

Snowling et al. (1998) realizaron una investigación longitudinal y observaron que las características de los disléxicos varían de primero a segundo año de primaria, mostrado un trastorno más persistente y progresivo en los disléxicos fonológicos; mientras los superficiales mantuvieron las diferencias con el grupo control, indicando más bien un retraso. De este modo se destaca que la diferencia entre los subtipos está en el grado de severidad del déficit de procesamiento fonológico.

Hemos encontrado también que los lectores retrasados del tipo superficial tienen niveles de ejecución peores en las pruebas que evalúan los procesos lectores que los lectores normales del los grupos control utilizados. Si consideramos, a pesar que su número es muy reducido ( $11,9 \%$ de la muestras de LR), que rinden peor que los lectores normales de igual nivel lector podríamos suponer que sus dificultades en el procesamiento ortográfico podrían ser causa de sus problemas de lectura, ya que si han logrado avanzar en el desarrollo las habilidades de procesamiento fonológico lo que los hace más eficientes en la lectura de pseudopalabras. Lo que a nosotros nos sugieren estos resultados es que los lectores retrasados de los años superiores han logrado cierto dominio de las reglas de conversión grafema fonema, lo que se ve facilitado por la características de nuestra sistema ortográfico, a costa de menores recursos cognitivos para disponer de las representaciones ortográficas de las palabras, así mismos sus dificultades en lectura reducen su experiencia con los textos escritos a pesar de sus años de escolarización, lo que no le ocurre a los lectores normales incluso de menor edad. De este modo los lectores retrasados del tipo superficial presentarian deficiencias en el procesamiento fonológico que podrían compensar con mayor tiempo de aprendizaje, mientras en los fonológicos su déficit es más estables. Estos resultados son consistentes con los datos obtenidos por Bruck $(1998,1992)$ con disléxicos universitarios.

En la última de las comparaciones, es decir la de los subtipos de lectores retrasados con lectores normales de igual grado escolar pero controlando estadísticamente el nivel lector, los análisis indican un número significativamente mayor de disléxicos fonológicos que de superficiales, $57,1 \%$ y $23,8 \%$ respectivamente. Comparando estos datos con los obtenidos de lectores retrasados en relación a lectores normales más jóvenes, tenemos que los muchos de los del subtipo retrasados (17) pasan a forma parte de los subgrupos fonológicos y superficiales.

Con este tipo de comparaciones se busca controlar la influencia de la experiencia lectora en el problema del retraso o desviación de los subtipos de lectores retrasados en el procesamiento lector. Los resultados nos muestran que la mayor parte de los disléxicos $(80,9 \%)$ presentan una disparidad importante entre lectura de pseudopalabras y discriminación ortográfica, lo que permite identificar un número significativo de disléxicos con un patrón del tipo fonológico y del tipo superficial. Se confirma la idea del déficit en el procesamiento fonológico de los disléxicos en general y de los fonológicos en particular, más allá de la experiencia lectora; la que si facilitaría el dominio de los procesos de recodificación fonológica, mejorando la exactitud en la lectura de pseudopalabras. Asimismo la experiencia lectora permitiría ir consolidando las representaciones ortográficas lo que podría explicar la disminución de los disléxicos moderados y de los superficiales, si relacionamos esta última comparación con los resultados de la realizada con los lectores normales de igual grado escolar.

Si tomamos como variable el tiempo de ejecución de las pruebas de lectura de pseudopalabras, observamos que en las tres comparaciones realizadas los lectores del subgrupo fonológico son más lentos que los grupos controles utilizado, lo que confirma el déficit fonológico que tiene, no sólo son menos exactos sino también más lentos. Si consideramos la comparación de los lectores retrasados con los lectores normales de igual grado escolar, el tiempo en lectura de pseudopalabras pone en evidencia el déficit fonológico de ambos subtipos: fonológicos y superficiales. 
Al tomar el tiempo en la prueba de discriminación ortográfica, en todas las comparaciones, los sujetos del tipo superficial necesitan de más tiempo que los fonológicos; y a su vez estos últimos no son sólo menos precisos, sino que requieren de un tiempo mayor que el de los grupos controles utilizados. Esto se puede explicar porque si bien los disléxicos fonológicos son más eficientes en el uso del procesamiento ortográfico que fonológico, presentan igualmente un procesamiento ortográfico deficiente. (Calvo, 1999).

Los disléxicos superficiales comparados con lectores normales de igual grado escolar se caracterizan también por la lentitud que tienen para leer. Esta baja velocidad sugiere una falta de automatización para el reconocimiento de palabras lo que incidirá luego en la capacidad de comprensión, como lo indican los trabajos de Wolf y Bowers (1999). Manis et al. (1999) también concluyeron en que la lentitud para leer es una característica más de la dislexia de superficie.

Las dificultades que hemos encontrado en los lectores retrasados del tipo superficial, sumado a la variabilidad en su distribución en las distintas comparaciones y lo deficiente que sigue siendo el procesamiento ortográfico de los fonológicos, nos sugiere por un lado la idea de que el reconocimiento visual sea secundario a la decodificación fonológica tal como lo plantean Aaron et al., (1999) y por otro lado la del continuo fonológico-superficial. Para estos últimos investigadores la habilidad para reconocer palabras de alta frecuencia surge gradualmente después del reconocimiento de las letras, por lo que se inicia primero un procesamiento fonológico para luego intentar el reconocimiento léxico; no habría entonces dos subtipos de dislexia sino dos etapas en reconocimiento de palabras cuyo déficit se reflejaría en estos subtipos de dislexia. Podría haber un continuo entre un subgrupo y otro en el que se identificarían caso extremos (Morais, 1998). Un continuo que se explica por la interdependencia y la sucesión, a través del desarrollo de la habilidad lectora, de los procesos de reconocimiento de palabras (Frith, 1985; Morais, 1998). Para lo cual también hay que considerar la transparencia ortográfica de la lengua y el tipo de enseñanza (Genard, 2000).

De esta forma las dislexia del tipo fonológico y superficial se diferenciarían en el gra- do de severidad del desorden fonológico y en los recursos cognitivo disponibles para compensar el déficit (Snowling, 2000). Los perfiles lectores de los sujetos con retraso lector depende de la severidad del déficit fonológico combinado con las variaciones en los recursos para el procesamiento en general, experiencia lectora y estrategias compensatorias (Griffith y Snowling, 2002). Lo que resulta compatible con nuestros datos, que muestran como sujetos con pobres ejecuciones en discriminación ortográfica son también poco eficientes en procesamiento fonológico. Así como también lo indican los resultados de Bailey et al. (2004), Castles et al. (1999) y Stanovich et al. (1997).

Esto se puede considerar además desde los planteos del autoaprendizaje de Share (1995, 1999), quien ha defendido la idea de que la recodificación fonológica funciona como un mecanismo de autoaprendizaje, permitiendo la adquisición de las representaciones ortográficas necesarias para el reconocimiento visual de las palabras rápido y autónomo. Cada decodificación correcta de una palabra no familiar, da la oportunidad de adquirir la información ortográfica precisa de la palabra, que es la base para un reconocimiento eficaz de las palabras. Un déficit fonológico interferirá primariamente en la lectura de pseudopalabras, pero puede también interferir en el mecanismo de autoaprendizaje que contribuye el reconocimiento directo de la palabra.

Al considerar los resultados en las pruebas metafonológicas, en los estudios realizados, nos encontramos que no hay en general diferencias significativas entre los distintos subtipos de lectores retrasados. Sólo se dieron diferencias entre los sujetos del subtipo fonológico y los del grupo control de igual grado escolar y lo de igual grado escolar controlado el nivel lector; en la prueba de omisión de sílaba inicial. Además de suponer que las pruebas elegidas no son las más adecuadas para discriminar a sujetos con retraso lector de $4^{\circ}$ a $6^{\circ}$ de primaria en relación a lectores normarles, diversos trabajos (Carrillo y Calvo, 1999; Santos, 1996, Wimmer, 1996. Porpodas, 1999; Goswami, 2002) plantea que los lectores retrasados de lenguas transparentes de los cursos superiores realizan con bastante exactitud las tareas metafonológicas, si bien pueden requerir más tiempo que los lectores normales. 


\section{REFERENCIAS}

Aaron P.G., Ayotolah M., Ellsberry A., Henderson J. y Lindsey K. (1999). Decoding an sight-word naming: Are they independent component of word recognition skill?. Reading an Writing: An Interdisciplinary Journal, 11:89-127.

Algarabel, S.(1996) Índices de interés psicolingüístico de 1.917 palabras castellanas. Cognitiva, 8 (1), 43-88

Asensio, M., (1989). Los Procesos De Lectura De Los Deficientes Auditivos. Tesis de doctorado. Universidad Autónoma de Madrid.

Bailey, C., Manis, F., Pedersen, W. y Seidenberg, M. (2004). Variation among developmental dyslexics: Evidence from a printed-word-learning task. Journal of Experimental Child Psychology. 87:125-154.

Calvo, A. (1999). Adquisición de la lectura en lengua castellana: perfiles cognitivos de aprendices con dificultades. Tesis de doctorado. Universidad de Murcia.

Carrillo, M., Marín, J. y Calvo, A. (1999). Distribución de subtipo de dislexia durante el desarrollo de la habilidad lectora. IV Simposio de Picolingüística. Madrid. 21 - 24 Abril.

Castles, A. \& Coltheart,M. (1993). Varieties of developmental dyslexia. Cognition, 47(2), 149-180

Cattell, R.B. y Cattell, A.K.S. (1950). Handbook for the individual or Group Culture Fair (or Free) Intelligence Test (a measure of " $g$ "). Scale 1. Illinois: Institute for Personality an Ability Testing. Publicado en castellano por TEA S.A. Madrid (1994).

Cuadro, A. (2006). Caracterización de los malos lectores en relación a los buenos lectores en niños uruguayos. Tesis doctoral. Universidad de Murcia. España

Cuetos, F., Rodríguez, B., y Ruano, E. (1998). PROLEC. Batería de Evaluación de los Procesos Lectores de los Niños de Educación Primaria. Madrid: TEA

Domínguez, A., De Vega, M. \& Cuetos,, F. (1997). Lexical inhibition from syllabic units in Spanish visual Word recognition. Language and Cognitive Processes, 12(4),401-422

Frith, U.(1985) Beneath the surface of developmental dyslexia. En K.E. Patterson, J.C.

Marshall y M. Coltheart (Eds.) Surface dyslexia: Neuropsychological and cognitive studies of phonological reading (pp.310-330) Hillsdale, NJ: Lawrence Erlbaum Associates. Genard (2000) La dyslexie du développement: étude de son caractère unique ou multiple de son étiologieà l'aide d'une approche comparative et logitudinale. Tesis Doctoral. Universidad Libre de Brusselas.
Jiménez, J. E., Álvarez, C., Estévez, A. y Hernández, I. (1998). Onset-Rime units in visual word recognition in normal Spanish readers and reading disabled children. Papers presented to I.A.R.L.D. Padua.

Manis, F. R., Seidenberg, M.S., Doi, L.,S., McBrideChang, C. \& Petersen, A. (1996). On the bases of two subtypes of development dyslexia. Cognition, 58, 157-195

Manis, F. R., Seidenberg, M, \& Doi, L., (1999). Rapid naming and the longitudinal prediction of reading sub-skills in first and second graders. Scientific Studies of reading, 3, 129-157

Marín, J. y Carrillo, M. S. (1999) Test Colectivo de Eficacia Lectora (TECLE).

Manuscrito no publicado. Departamento de Psicología Básica y Metodología. Universidad de Murcia.

Marín, J., Aveledo, F., Vera, F. y Alegría, J. (2004). El papel de la fonología en la adquisición del léxico ortográfico. El caso de los grafemas "g" y "c". Comunicación presentada al V Congreso de la Sociedad Española de Psicología Experimental (SEPEX) Madrid.

Morais, J. (1998). El Arte De Leer, Madrid, Visor.

Porpodas, C. (1999) Patterns of phonological and memory processing in beginning readers and spellers of Greek. Journal of Learning Disabilities. Vol 32, Issue 5, 384

Santos, A. (1996). Intervención En Dificultades En Lectoescritura Para $2^{\circ}$ De Educación Primaria. En servicio municipal de atención psicológica y educativa: Memoria De Actividades, 1994/95, Patronato Municipal De Servicios/Ayuntamiento De San Sebastián De Los Reyes (Madrid).

Seymour, P.H.K. \& MacGrecor, C. J.(1984) Developmental dyslexia: a cognitive experimental analysis of phonological, morphemic and visual impairments. Cognitive Neuropsychology, 1,43-83

Seymour, P.H.K., \& Evans, H. M. (1993). The visual (orthographic) processor and developmental dyslexia. In D.M. Willows, R.S. Kruk, \& E. Crocos (Eds), Visual processes in reading and reading disabilities (pp.347-376) Hillsdale, New Jersey: LEA, Hove and London.

Share, D. L. (1995) Phonological recoding and selfteaching: sine qua non of reading acquisition. Cognition, 55, 515-218.

Share, D. L. (1999). Phonological recoding and orthographic learning: A direct test of the selfteaching hypothesis. Journal of Experimental Child Psychology 72, 95129

Share, D. L.; Stanovich, K. (1995) Cognitive processes in early reading development; Accommodating 
individual differences into a model of acquisition. Issues in Education, 1, 1-57.

Snowling, M. J. (1998).Dyslexia. Oxford. England: Blackwell

Snowling, M. J., Goulandris, N., \& Defty, N. (1998). Development and variation in developmental dyslexia. In C. Hulme \& J.R. Malatesha (Eds.) Reading and spelling: Development and disorders (pp. 201-217). Mahwah, NJ, USA: Lawrence Erlbaum Associates.

Stanovich, K. E., Siegel, L. S., \& Gottardo, A. (1997). Converging evidence for phonological and surface subtypes of reading disability. Journal of Educational Psychology, 89(1), 114-127.

Wimmer H. (1993). Characteristics of developmental dyslexia in a regular writing system. Applied Psycholinguistics, 14 1-33.

Wimmer, H. (1996b) The non-word reading deficit in developmental dyslexia: Evidence from children learning to read German. Journal of Experimental Child Psychology, 61, 80-90.

Wolf, M., \& Bowers, P. G. (1999). The double-deficit hypothesis for the developmental dyslexias. Journal of Educational Psychology, 91(3), 415-438. 